\title{
Assessment of biosecurity measures in broiler's farms in the Suez Canal area - Egypt using a seasonal prevalence of Salmonellosis
}

\author{
Essam S. Soliman ${ }^{1}$ iD and Mona S. Abdallah² \\ 1. Department of Animal Hygiene, Zoonosis, and Animal Behavior, Faculty of Veterinary Medicine, Suez Canal University, \\ Ismailia, 41522, Egypt; 2. Department of Avian and Rabbit Medicine, Faculty of Veterinary Medicine, Suez Canal \\ University, Ismailia, 41522, Egypt. \\ Corresponding author: Essam S. Soliman, e-mail: soliman.essam@vet.suez.edu.eg \\ Co-author: MSA: drmona_salim@yahoo.com \\ Received: 12-11-2019, Accepted: 28-02-2020, Published online: 08-04-2020
}

doi: www.doi.org/10.14202/vetworld.2020.622-632 How to cite this article: Soliman ES, Abdallah MS (2020) Assessment of biosecurity measures in broiler's farms in the Suez Canal area - Egypt using a seasonal prevalence of Salmonellosis, Veterinary World, 13(4): 622-632.

\begin{abstract}
Background and Aim: Biosecurity practices are a must in broiler farms to reduce the risk of infectious agents. This study aimed to evaluate biosecurity measures in nine broiler farms in the Suez Canal area - Egypt with measuring the seasonal prevalence of salmonellosis.

Materials and Methods: A cross-sectional study was conducted on randomly selected nine broiler farms of different housing systems based on the ventilation methods from March 2018 to April 2019. A total of 12,600 samples (6480 environmental, 4320 non-environmental, 1080 sera, and 720 live birds) were collected during four successive seasons.

Results: Highly significant increases $(\mathrm{p}<0.01)$ were recorded in body weight gains in opened and closed-houses during summer; in food conversion ratios in opened-houses during winter and in closed-houses during winter and fall; in performance indices in opened-houses during summer and closed-houses during winter; and in live body weights, carcasses weights, liver, spleen, and bursa's weights in opened-houses during spring and in closed-houses during fall. Highly significant increases $(p<0.01)$ were recorded in total bacterial, Enterobacteriaceae, and Salmonella counts in opened-houses during spring and in closed-houses during summer, in Salmonella Typhi O and H, and Salmonella Paratyphi A and B sera titer in opened-houses during summer and closed-houses during fall. Biosecurity measures scored 34 out of 43 with an average salmonellosis prevalence of $6.0 \%$ in closed-house and 24 out of 43 with an average salmonellosis prevalence of $24.67 \%$ in opened-house broiler farms.
\end{abstract}

Conclusion: Weak biosecurity measures in broiler houses (opened and closed) were not sufficient to prevent the entrance and multiplication of Salmonella spp. Disciplines, commitment, and regulations of biosecurity need to be enforced in broiler houses to prevent the introduction and spread of diseases.

Keywords: biosecurity, broiler, Egypt, housing systems, Salmonella, seasons.

\section{Introduction}

Biosecurity can be described as principles, measures, and strategies that should be taken to prevent the introduction of a causative agent into a farm known as bioexclusion and to prevent the spread of a causative agent from one farm to another known as bioconfinement and biocontainment, as well as to prevent the spread of a causative agent inside the farm from one area to another known as biomanagement [1]. Successful biosecurity program depends initially on marking out boundaries of the production area inside the farm to ensure secure boundaries which represent the first line of defense against the introduction of infectious diseases [2]. Biosecurity programs in poultry farms should also consider some parameters,

Copyright: Solimanand and Abdallah. Open Access. This article is distributed under the terms of the Creative Commons Attribution 4.0 International License (http://creativecommons.org/licenses/ by/4.0/), which permits unrestricted use, distribution, and reproduction in any medium, provided you give appropriate credit to the original author(s) and the source, provide a link to the Creative Commons license, and indicate if changes were made. The Creative Commons Public Domain Dedication waiver (http:// creativecommons.org/publicdomain/zero/1.0/) applies to the data made available in this article, unless otherwise stated. including the number of houses in each farm, the distance between houses, houses location and design, stocking density, interior arrangement, ventilation system, as well as, human, food, and material movement throughout the farm.

Poultry production depends on intensification and housing in closed confinement, which facilitates disease spread rapidly from one farm to another during an outbreak with economic devastation [3,4]. Inadequate biosecurity measures, absence and/or weak disease control measures and strategies [5], and poor management practices [6] contribute to high mortalities in poultry farms due to direct or indirect introduction and spread of some infectious and contagious pathogens such as infectious bursal disease (IBD) virus, Newcastle disease (ND) virus, and Salmonella spp. causing fowl typhoid and salmonellosis [7].

Salmonella Enteritidis normally inhabits the intestinal tract of birds, excreted in feces for months, and transmitted by insect, rodent, equipment, human being, soil, air, and water [8,9]. Once infection stabilized in a productive flock, it can transmit vertically and horizontally to infect other farms easily with zoonotic 
potential, especially Salmonella Typhimurium and $S$. Enteritidis [10]. The environment can act as a source of infection for salmonellosis, as Salmonella spp. can survive in the environment for a long period [11]. Salmonella spp. serological identification depends on using some commercial polyvalent antisera kits that contained a mixture of antibodies for determining the somatic $(\mathrm{O})$ and flagellar $(\mathrm{H})$ antigens [12].

The study aimed at evaluating the variable biosecurity measures installed in nine broiler farms with different housing systems based on the ventilation methods used (naturally ventilated or opened and artificially ventilated or closed-houses) in the Suez Canal area - Egypt using the seasonal prevalence of salmonellosis as an indicator for the successful application of these measures.

\section{Materials and Methods}

\section{Ethical approval}

The current protocol was reviewed and approved by the Scientific Research Ethics Committee of the Faculty of Veterinary Medicine, Suez Canal University, Ismailia, Egypt, with approval number (2019028).

\section{Study design}

A cross-sectional study was conducted by visiting nine broiler farms in the Suez Canal area - Egypt. The nine farms were selected using systemic random sampling procedures, as recommended by Bell House [13]. Targeted farms' selection was based on their geographical location in the three governorates overlook the Suez Canal area - Egypt (Port Said, Ismailia, and Suez governorates). Visits were carried out weekly for 13 months (March 2018-April 2019). Farms under study were raising three different broiler breeds; are Ross 306 (in four farms, one located in Port Said, two located in Ismailia, and one farm located in Suez governorate), Cobb 500 (in two farms both located in Port Said governorate), and Hubbard classic broilers (in three farms, one located in Ismailia, and two farms located in Suez governorate).

Broilers in the nine farms were brooded and housed at optimal microclimatic temperature (received at $35^{\circ} \mathrm{C}$, decreased gradually until a stable $24-26^{\circ} \mathrm{C}$ was achieved by the end of the $3^{\text {rd }}$ week with the latter comfort zone of $21-24^{\circ} \mathrm{C}$ ). Broilers in the nine farms were supplied with a common continuous lighting regimen (23 $\mathrm{h}$ lighting and $1 \mathrm{~h}$ darkness) using white LED lights, as recommended by Soliman and Hassan [14]. Birds in the nine farms were given ad libitum access to drinking water and supplied with corn and soybean diet of the same constituents (El-Eman Co, El Sharkia, Egypt). The rations supplied to the broilers in the nine farms were containing $23 \%$ protein, $4.81 \%$ fat, $3.3 \%$ crude fiber, and $3000 \mathrm{kcal} / \mathrm{kg}$ energy in the starter ration and $21 \%$ protein, $5.89 \%$ fat, $3.5 \%$ crude fiber, and $3100 \mathrm{kcal} / \mathrm{kg}$ energy in the grower ration, as recommended by NRC [15] and Applegate and Angel [16].

Each farm followed a selective vaccination program, with a common baseline vaccination of birds against infectious bronchitis, IBD, and ND. The location, broilers' breeds, housing system, number of units per farm, bird's capacity in each unit, ventilation system and mechanism, feeding and watering systems, and lighting durations of each farm are listed in Table-1.

\section{Biosecurity evaluation and scoring system}

Biosecurity measures in each of the nine farms were evaluated eventually according to certain characteristics as set by Wei and Aengwanich [17], including the following: Attraction of wild birds, prevention of wild birds, measures for farmworkers, measures for new poultry on the farms, measures for farm visitors, measures for vendors, measures for equipment and vehicles, water sources and water quality care, food sources, and environments surrounding farms including the distance between farms and roads, and other parts, type of poultry on farms, cleaning, and disinfection capacity on farms, measures from entry to poultry pens, and biosecurity system planning inside farms.

The nine farms that were previously identified and highlighted for regular weekly visits were evaluated and scored for each of the listed 14 characteristics according to a scale from 0 to 3 , creating a top score of 43. The scoring was carried out weekly during the visits in reference to the compatibility of the interior arrangements and procedures enforced inside each farm regarding the characteristics set by Wei and Aengwanich [17] and was used for evaluation. Final average scores were calculated out of the values recorded during the 13 months for each farm in reference to all the characteristics used for evaluations.

Table-1: Broiler farms' basic information in the farms under evaluation for biosecurity measures.

\begin{tabular}{|c|c|c|c|c|c|c|c|c|}
\hline $\begin{array}{l}\text { Farm } \\
\text { no. }\end{array}$ & Location & $\begin{array}{l}\text { Broiler's } \\
\text { breed }\end{array}$ & $\begin{array}{l}\text { Housing } \\
\text { system }\end{array}$ & $\begin{array}{c}\text { No. of } \\
\text { units/Farm }\end{array}$ & $\begin{array}{c}\text { Bird capacity/ } \\
\text { Unit }\end{array}$ & $\begin{array}{l}\text { Ventilation } \\
\text { system }\end{array}$ & $\begin{array}{l}\text { Feed and } \\
\text { watering system }\end{array}$ & $\begin{array}{l}\text { Lighting } \\
\text { program }\end{array}$ \\
\hline 1 & Port said & Cobb & Deep litter & 3 & 10000 & Natural - Cross & Manual & 23L:1D \\
\hline 2 & Ismailia & Ross & Deep litter & 5 & 5000 & Natural - Cross & Manual & 23L:1D \\
\hline 3 & Ismailia & Hubbard & Deep litter & 5 & 5000 & Natural - Cross & Manual & 23L:1D \\
\hline 4 & Port Said & Cobb & Battery & 5 & 10000 & Natural - Cross & Manual & 23L:1D \\
\hline 5 & Suez & Hubbard & Deep litter & 10 & 20000 & Automated-Tunnel & Automated & 23L:1D \\
\hline 6 & Ismailia & Ross & Deep litter & 5 & 10000 & Natural-Cross & Manual & 23L:1D \\
\hline 7 & Suez & Hubbard & Slatted floor & 20 & 20000 & Automated-Tunnel & Automated & 23L:1D \\
\hline 8 & Suez & Ross & Slatted floor & 20 & 20000 & Automated-Tunnel & Automated & 23L:1D \\
\hline 9 & Port Said & Ross & Battery & 5 & 10000 & Natural-Cross & Manual & 23L:1D \\
\hline
\end{tabular}


The average means of salmonellosis (mean $\pm \mathrm{SE}$ ) are displayed in Table-2 in relation to the different housing systems, and seasonal variation along with their interactions, as well as average scores of biosecurity measures for each farm are displayed in Table-3.

\section{Sampling}

A total of 12600 samples were collected during the four seasons from the nine farms, including 6480 environmental samples (litter, water, walls, fans, feeders, and waterers swabs), 4320 non-environmental samples (liver, spleen, duodenum of the intestine, and breast muscle), 1080 blood samples for sera separation, and 720 live birds were collected by the end of the studied cycles for carcass quality evaluation. Blood samples were transferred to the laboratory in a dry ice box and centrifuged at $2500 \mathrm{rpm}$ for $20 \mathrm{~min}$ for sera separation. Clear non-hemolyzed sera were stored at $-20^{\circ} \mathrm{C}$ until used for the serological identification of Salmonella spp.

Birds collected for carcass evaluation were transported using conditioned trucks. On their arrival, birds were rested for $3 \mathrm{~h}$ then slaughtered in the institutional experimental slaughter room. Liver, spleen, heart, and bursa were extracted, weighed, and expressed as $(\mathrm{g} / \mathrm{kg})$, and the carcasses were weighed after de-feathering and evisceration and expressed as (g), as recommended by Soliman et al. [18]. The carcasses were hygienically disposed of after weighing and sampling using burial technique in reference to the lining of the burial bits with lime.
Environmental swabs, as well as, non-environmental samples were collected and placed in $9 \mathrm{ml}$ buffered peptone water, preserved in a dry ice box supplied with gel bags to maintain the samples and retard any biological changes, and transferred to the laboratory for bacteriological assessment considering a transportation time that did not exceed 2-3 $\mathrm{h}$.

\section{Performance indices (PI)}

Live body weights (LBW) expressed by grams (g) were measured based on weighing representative samples of birds in each farm, the representative sample sizes were calculated using a simple random sampling design [19] with an expected error 5\% using the following formula:

$$
\mathrm{n}=1.96^{2} \mathrm{P}_{\text {exp }}\left(1-\mathrm{P}_{\exp }\right) / \mathrm{d}^{2}
$$

Where $n=$ required sample size, $\mathrm{P}_{\exp }=$ expected prevalence, and $\mathrm{d}=$ desired absolute precision. Approximately 286 out of each 1000 birds in each farm were weighed to obtain representative and accurate measures. Feed intakes (FI, expressed by g) were calculated by dividing the total amounts of ration consumed by birds in each building by the actual number of birds housed in this building. Bodyweight gains (BWG, expressed by g), feed conversion ratio (FCR, expressed by \%), and (PI, expressed as a ratio) were calculated, as recommended by Soliman and Hassan [20].

Table-2: Salmonella Serotypes titer (Mean \pm SE) in sera collected from broilers raised on different housing systems during different seasons.

\begin{tabular}{|c|c|c|c|c|c|}
\hline \multirow{2}{*}{$\begin{array}{l}\text { Housing } \\
\text { system }\end{array}$} & \multicolumn{4}{|c|}{ Salmonella Serotype titer } & \multirow{2}{*}{$\begin{array}{c}\text { Salmonella } \\
\text { prevalence \% }\end{array}$} \\
\hline & $\mathbf{0}$ & $\mathbf{H}$ & $\mathbf{A H}$ & BH & \\
\hline \multicolumn{6}{|c|}{ Overall means of the housing system } \\
\hline Opened-house & $157.2^{\mathrm{a}} \pm 2.83$ & $163.9^{\mathrm{a}} \pm 2.99$ & $110.6^{\mathrm{a}} \pm 1.44$ & $113.6^{\mathrm{a}} \pm 1.57$ & $24.6^{\mathrm{a}} \pm 0.45$ \\
\hline Closed-house & $104.8^{\mathrm{b}} \pm 4.01$ & $104.6^{b} \pm 4.23$ & $81.1^{\mathrm{b}} \pm 2.03$ & $80.0^{b} \pm 2.23$ & $6.0^{\mathrm{b}} \pm 0.05$ \\
\hline $\mathrm{p}$-value & 0.016 & 0.009 & 0.001 & 0.000 & 0.001 \\
\hline \multicolumn{6}{|c|}{ Overall means of the seasons } \\
\hline Summer & $141.8^{\mathrm{a}} \pm 2.91$ & $138.1^{\mathrm{a}} \pm 5.12$ & $100.4^{\mathrm{a}} \pm 2.49$ & $105.7^{\mathrm{a}} \pm 2.73$ & $15.6^{\mathrm{a}} \pm 0.06$ \\
\hline Fall & $133.8^{\mathrm{a}} \pm 2.88$ & $138.7^{\mathrm{a}} \pm 3.17$ & $93.3^{\mathrm{a}} \pm 1.94$ & $93.1^{\mathrm{a}} \pm 1.55$ & $13.8^{b} \pm 0.12$ \\
\hline Winter & $127.1^{\mathrm{b}} \pm 3.46$ & $132.4^{\mathrm{a}} \pm 5.18$ & $95.3^{a} \pm 1.66$ & $96.0^{a} \pm 2.34$ & $9.6^{c} \pm 0.08$ \\
\hline Spring & $121.4^{b} \pm 2.65$ & $127.8^{\mathrm{a}} \pm 4.14$ & $94.4^{\mathrm{a}} \pm 1.93$ & $92.4^{\mathrm{a}} \pm 3.22$ & $5.2^{\mathrm{d}} \pm 0.06$ \\
\hline $\mathrm{p}$-value & 0.025 & 0.516 & 0.624 & 0.725 & 0.002 \\
\hline \multicolumn{6}{|c|}{ Housing system versus seasonal variation interactions } \\
\hline \multicolumn{6}{|l|}{ Opened-house } \\
\hline Summer & $177.0^{\mathrm{a}} \pm 7.17$ & $174.0^{\mathrm{a}} \pm 6.97$ & $119.1^{\mathrm{a}} \pm 4.15$ & $131.5^{\mathrm{a}} \pm 5.33$ & $29.2^{\mathrm{a}} \pm 0.08$ \\
\hline Fall & $152.2^{b} \pm 6.35$ & $161.1^{\mathrm{b}} \pm 6.68$ & $106.6^{b} \pm 3.56$ & $106.2^{\mathrm{c}} \pm 3.01$ & $28.6^{\mathrm{a}} \pm 0.02$ \\
\hline Winter & $150.2^{b} \pm 6.29$ & $158.2^{b} \pm 6.78$ & $110.6^{b} \pm 3.10$ & $112.0^{\mathrm{b}} \pm 3.48$ & $22.0^{\mathrm{b}} \pm 0.01$ \\
\hline Spring & $149.5^{b} \pm 6.67$ & $162.3^{b} \pm 7.32$ & $106.2^{\mathrm{b}} \pm 3.01$ & $104.8^{c} \pm 3.16$ & $19.3^{c} \pm 0.05$ \\
\hline p-value & 0.092 & 0.182 & 0.123 & 0.002 & 0.000 \\
\hline \multicolumn{6}{|l|}{ Closed-house } \\
\hline Summer & $106.6^{b} \pm 3.99$ & $102.2^{b} \pm 3.79$ & $81.7^{\mathrm{a}} \pm 1.24$ & $80.0^{\mathrm{a}} \pm 0.00$ & $9.5^{\mathrm{a}} \pm 0.03$ \\
\hline Fall & $115.5^{\mathrm{a}} \pm 5.22$ & $116.4^{\mathrm{a}} \pm 6.08$ & $80.0^{\mathrm{a}} \pm 0.00$ & $80.0^{\mathrm{a}} \pm 0.00$ & $5.0^{\mathrm{b}} \pm 0.01$ \\
\hline Winter & $104.0^{\mathrm{b}} \pm 3.88$ & $106.6^{b} \pm 5.05$ & $80.0^{\mathrm{a}} \pm 0.00$ & $80.0^{\mathrm{a}} \pm 0.00$ & $5.3^{b} \pm 0.02$ \\
\hline Spring & $93.3^{c} \pm 3.16$ & $100.3^{b} \pm 3.16$ & $82.6^{a} \pm 0.00$ & $80.0^{\mathrm{a}} \pm 0.00$ & $4.3^{b} \pm 0.01$ \\
\hline p-value & 0.023 & 0.098 & 0.325 & 0.815 & 0.000 \\
\hline
\end{tabular}

Means carrying different superscripts in the same column are significantly different at $(\mathrm{p} \leq 0.05)$ or highly significantly different at $(p<0.01)$. Means carrying the same superscripts in the same column are non-significantly different at $(\mathrm{p}<0.05) . \mathrm{O}=$ Salmonella Typhi $\mathrm{O}, \mathrm{H}=$ Salmonella Typhi $\mathrm{H}, \mathrm{AH}=$ Salmonella Paratyphi A, BH=Salmonella Paratyphi B, opened=naturally ventilated houses, Closed=artificially ventilated houses, SE=Standard error 
Table-3: The evaluation system for the biosecurity programs and the prevalence of salmonellosis in farms under study.

\begin{tabular}{|c|c|c|c|c|c|c|c|c|c|}
\hline Evaluation items & $\mathbf{F 1}$ & $\mathbf{F 2}$ & F3 & F4 & $\mathbf{F 5}$ & F6 & F7 & F8 & F9 \\
\hline Av. Prevalence of salmonellosis \% & 29 & 25 & 27 & 23 & 5 & 18 & 7 & 6 & 26 \\
\hline Attraction of wild birds & 3 & 2 & 2 & 1 & 0 & 3 & 0 & 0 & 2 \\
\hline Prevention of wild birds & 0 & 1 & 2 & 2 & 3 & 0 & 3 & 3 & 2 \\
\hline Measures for farm workers & 1 & 1 & 1 & 2 & 2 & 1 & 1 & 2 & 2 \\
\hline Measures for new poultry on the farms & 2 & 2 & 1 & 2 & 3 & 2 & 3 & 3 & 3 \\
\hline Measures for farm visitors & 1 & 2 & 1 & 2 & 3 & 1 & 3 & 3 & 2 \\
\hline Measures for vendors & 1 & 2 & 1 & 2 & 3 & 1 & 2 & 2 & 1 \\
\hline Measures for equipment and vehicles & 1 & 2 & 2 & 2 & 2 & 1 & 2 & 2 & 2 \\
\hline Water sources and water quality care & 2 & 2 & 2 & 3 & 3 & 2 & 3 & 3 & 2 \\
\hline Food sources & 1 & 2 & 2 & 3 & 3 & 1 & 3 & 3 & 2 \\
\hline $\begin{array}{l}\text { Environments surrounding farms: Distance } \\
\text { between farms and roads, and other parts }\end{array}$ & 2 & 2 & 1 & 2 & 3 & 2 & 3 & 3 & 2 \\
\hline Type of poultry on farms & 1 & 2 & 2 & 2 & 3 & 1 & 3 & 3 & 3 \\
\hline Cleaning and disinfection capacity on farms & 2 & 2 & 1 & 2 & 2 & 2 & 2 & 3 & 2 \\
\hline Measures from entry to poultry pens & 2 & 2 & 2 & 2 & 3 & 2 & 3 & 2 & 2 \\
\hline Biosecurity system planning inside farms & 1 & 2 & 2 & 2 & 2 & 1 & 2 & 3 & 2 \\
\hline Total score out of 43 & 20 & 26 & 22 & 29 & 35 & 20 & 33 & 35 & 29 \\
\hline
\end{tabular}

Zero-point was the lowest score and 3 points were the highest score for each point out of the 14 points for evaluating the biosecurity measures, F=farm. F1, F2, F3, F4, F6, and F9 are naturally ventilated (opened-house) farms with an average biosecurity score of 24 out of 43 and average salmonellosis prevalence $24.67 \%$; F5, F7, and F8 are artificially ventilated (closed-house) farms with an average score of 34 out of 43 and average salmonellosis prevalence $6.0 \%$

\section{Serological analysis of Salmonella spp.}

Sera were used in a serological quantitative antigen-antibody micro-well agglutination test [21,22]. The test aims at detecting Salmonella Typhi immunoglobulin $\mathrm{M}$ against the somatic antigen $(\mathrm{O})$, flagellar antigen $(\mathrm{H})$, Salmonella Paratyphi A (AH), and $S$. Paratyphi B (BH). The degree of agglutination was observed and quantified.

\section{Bacteriological examination}

Litter samples were prepared by weighing and adding $3 \mathrm{~g}$ into $27 \mathrm{ml}$ physiological saline and filtered, as recommended by Soliman et al. [23]. Nonenvironmental organs such as the liver, spleen, duodenum of the intestine, and breast muscles were added to $0.1 \%$ sterile buffered peptone water, homogenized using a stomacher (Lab. Blender 400, Seward Lab., London). Litter filtrate, water samples, environmental swabs, and non-environmental homogenized extract were prepared according to APHA [24] using ten-fold serial dilutions up to $10^{-6}$ to cover the expected range of samples contamination, which could be easily counted.

Total bacterial count (TBC) onto standard plate count agar (SPC, APHA dehydrated Thermo Scientific $^{\mathrm{TM}}$ Oxoid $^{\mathrm{TM}}$ CM0463B, weight $500 \mathrm{~g}$ ) at $37^{\circ} \mathrm{C}$ for $24 \mathrm{~h}$, total Enterobacteriaceae count (TEC) onto Eosin methylene blue agar (EMB, Modified Levine Thermo Scientific ${ }^{\mathrm{TM}}$ Oxoid ${ }^{\mathrm{TM}}$ CM0069B, weight $500 \mathrm{~g}$ ) at $37^{\circ} \mathrm{C}$ for $24 \mathrm{~h}$ for detecting the ideal metallic green colonies, and total Salmonella count (TSC) onto CHROMagar ${ }^{\mathrm{TM}}$ Salmonella (BD BBL $^{\mathrm{TM}}$ CHROMagar ${ }^{\mathrm{TM}}$ Salmonella READY-TO-USE Plated Media) at $37^{\circ} \mathrm{C}$ for $24 \mathrm{~h}$ for detecting the ideal pink colonies were applied using drop plate technique, according to Kim and Lee [25]. Plates showed that 30-300 colony-forming units (CFU) were counted using the Darkfield colony counter (R164109 Reichert-Jung Quebec Darkfield 3325 Colony Counter) [26].

\section{Statistical analysis}

Statistical analysis was carried out using the Statistical Package for the Social Sciences (SPSS), IBM SPSS statistics version-20 [27]. The obtained data were analyzed statistically using multifactorial Analysis of Variance (Two-way ANOVA) investigating the effects of the housing systems based on ventilation methods and seasons along with their interactions. The interaction effects of the housing systems (closed and opened) and the seasons (summer, fall, winter, and spring) were displayed in the tables along with the overall and main effects. The statistical model was summarized as follow:

$$
\mathrm{Y}_{\mathrm{ijk}}=\mu+\alpha_{\mathrm{i}}+\beta_{\mathrm{j}}+(\alpha \beta)_{\mathrm{ij}}+\mathrm{e}_{\mathrm{ijk}}
$$

Where $Y_{\mathrm{ijk}}$ was the measurement of the dependent variables; $\mu$ was overall mean; $\alpha_{i}$ was the fixed effect of the housing system; $\beta_{\mathrm{j}}$ was the fixed effect of the season; $(\alpha \beta)_{\mathrm{ij}}$ was the interaction effect of the housing systems by seasons; $\mathrm{e}_{\mathrm{ijk}}$ was the random error. The significant levels were expressed as highly significant at $\mathrm{p}<0.01$, significant at $\mathrm{p} \leq 0.05$, and non-significant at $\mathrm{p}>0.05$. The bacterial counts were transferred into logarithmic numbers as well as, the average scores for the nine farms understudy were calculated using Microsoft Excel 2016.

\section{Results}

\section{Performance traits}

Increases in weight gains and PI of broilers raised in closed-houses were highly significant $(\mathrm{p}<0.01)$ and the increases in FI and FCRs of broilers raised in opened-houses were also highly significant $(\mathrm{p}<0.01$; Table-4), there were no significant differences in the weight gains among the four seasons (Table-4). With regard to $F I$, highly significant increases $(p<0.01)$ were observed during winter, spring, fall, and 
Table-4: Performance traits (Mean \pm SE) in broilers raised on different housing systems during different seasons.

\begin{tabular}{|c|c|c|c|c|}
\hline \multirow[t]{2}{*}{ Housing system } & \multicolumn{4}{|c|}{ Performance traits } \\
\hline & $\mathbf{W G} / \mathbf{g}$ & $\mathbf{F I} / \mathbf{g}$ & FCR (\%) & PI \\
\hline \multicolumn{5}{|c|}{ Overall means of the housing system } \\
\hline Opened-house & $341.7^{b} \pm 2.91$ & $557.7^{a} \pm 0.95$ & $1.66^{\mathrm{a}} \pm 0.01$ & $5.30^{\mathrm{b}} \pm 0.06$ \\
\hline Closed-house & $397.1^{\mathrm{a}} \pm 4.11$ & $463.1^{\mathrm{b}} \pm 1.34$ & $1.20^{\mathrm{b}} \pm 0.02$ & $8.57^{\mathrm{a}} \pm 0.09$ \\
\hline p-value & 0.001 & 0.005 & 0.002 & 0.000 \\
\hline \multicolumn{5}{|c|}{ Overall means of the seasons } \\
\hline Summer & $378.0^{\mathrm{a}} \pm 1.22$ & $495.4^{d} \pm 1.62$ & $1.34^{\mathrm{c}} \pm 0.02$ & $7.40^{\mathrm{b}} \pm 0.11$ \\
\hline Fall & $369.0^{\mathrm{a}} \pm 2.13$ & $506.5^{c} \pm 0.93$ & $1.42^{\mathrm{b}} \pm 0.01$ & $6.93^{c} \pm 0.09$ \\
\hline Winter & $361.6^{\mathrm{a}} \pm 1.33$ & $526.1^{a} \pm 0.99$ & $1.53^{\mathrm{a}} \pm 0.01$ & $6.50^{`} \pm 0.08$ \\
\hline Spring & $369.1^{\mathrm{a}} \pm 2.11$ & $513.5^{\mathrm{b}} \pm 1.22$ & $1.43^{\mathrm{b}} \pm 0.01$ & $9.63^{a} \pm 0.12$ \\
\hline p-value & 0.532 & 0.001 & 0.000 & 0.023 \\
\hline \multicolumn{5}{|c|}{$\begin{array}{l}\text { Housing system versus seasonal variation interactions } \\
\text { Opened-house }\end{array}$} \\
\hline Summer & $352.8^{\mathrm{a}} \pm 4.23$ & $531.8^{d} \pm 1.90$ & $1.52^{\circ} \pm 0.02$ & $5.91^{a} \pm 0.13$ \\
\hline Fall & $341.6^{b} \pm 5.82$ & $548.7^{c} \pm 2.23$ & $1.62^{\mathrm{b}} \pm 0.03$ & $5.34^{b} \pm 0.21$ \\
\hline Winter & $330.5^{c} \pm 2.31$ & $584.6^{\mathrm{a}} \pm 3.21$ & $1.82^{\mathrm{a}} \pm 0.02$ & $4.71^{c} \pm 0.31$ \\
\hline Spring & $342.1^{b} \pm 5.66$ & $565.7^{b} \pm 0.98$ & $1.67^{b} \pm 0.02$ & $5.25^{b} \pm 0.14$ \\
\hline p-value & 0.037 & 0.000 & 0.010 & 0.038 \\
\hline \multicolumn{5}{|l|}{ Closed-house } \\
\hline Summer & $403.2^{\mathrm{a}} \pm 8.23$ & $459.0^{\mathrm{a}} \pm 2.21$ & $1.15^{\mathrm{b}} \pm 0.03$ & $8.89^{a} \pm 0.19$ \\
\hline Fall & $396.4^{\mathrm{b}} \pm 6.21$ & $464.3^{a} \pm 2.69$ & $1.21^{\mathrm{a}} \pm 0.04$ & $8.52^{\mathrm{a}} \pm 0.22$ \\
\hline Winter & $392.8^{b} \pm 5.41$ & $467.7^{a} \pm 2.65$ & $1.24^{\mathrm{a}} \pm 0.04$ & $8.28^{b} \pm 0.17$ \\
\hline Spring & $396.2^{b} \pm 4.32$ & $461.3^{a} \pm 3.41$ & $1.19^{\mathrm{ab}} \pm 0.02$ & $8.61^{\mathrm{a}} \pm 0.19$ \\
\hline p-value & 0.867 & 0.662 & 0.056 & 0.223 \\
\hline
\end{tabular}

Means carrying different superscripts in the same column are significantly different at $(p \leq 0.05)$ or highly significantly different at $(p<0.01)$. Means carrying the same superscripts in the same column are non-significantly different at $(p<0.05) . W G=$ Weight Gain, FI=Feed Intake, FCR=Feed Conversion Ratio, PI=Performance Index, Opened=naturally ventilated houses, Closed=artificially ventilated houses, $\mathrm{SE}=$ Standard error

summer, respectively (Table-4). Increases in FCRs were highly significant $(\mathrm{p}<0.01)$ as observed during winter, fall, spring, and summer, respectively, with no significant differences between FCRs during fall and spring seasons (Table-4). PI revealed highly significant increases $(p<0.01)$ during spring, summer, fall, and winter seasons, respectively, with no significant differences between PI during fall and winter seasons (Table-4).

BWGs interactions, as recorded in Table-4, showed highly significant increases $(p<0.01)$ during summer in both opened- and closed-house broiler farms. FIs interactions revealed, in Table-4, highly significant increases $(\mathrm{p}<0.01)$ during winter in openedhouses, and no significant differences were recorded during all seasons in closed-house broiler farms.

FCRs' interactions recorded highly significant increases $(p<0.01)$ during winter in opened-house broiler farms, and during winter and fall with no significant difference in closed-house broiler farms (Table-4). Meanwhile, the PI interactions in Table-4 revealed highly significant increases $(p<0.01)$ during summer and winter in opened- and closed-house broiler farms, respectively.

\section{Carcass quality}

Overall means revealed in Table-5, highly significant increases $(\mathrm{p}<0.01)$ of LBW, carcass weights, spleen, and heart ratios in closed-house broiler farms, highly significant increases $(p<0.01)$ of liver ratios in opened-house broiler farms, and no significant differences between the two housing systems in bursa's ratios. LBW and heart ratios (Table-5) revealed highly significant increases $(\mathrm{p}<0.01)$ during fall, spring, summer, and winter seasons, respectively. Carcasses weights, spleen, and bursa ratios in Table- 5 revealed highly significant increases $(p<0.01)$ during fall, spring, summer, and winter, respectively, with no significant differences between carcasses weights during fall and spring seasons. The liver ratios (Table-5) revealed highly significant increases $(p<0.01)$ during spring, fall, summer, and winter seasons, respectively.

LBW, carcasses weights, liver; spleen, and bursa's ratios recorded highly significant increases $(\mathrm{p}<0.01)$, as shown in Table-5, during spring and fall seasons in opened- and closed-house broiler farms, respectively. The heart ratios revealed highly significant increases $(p<0.01)$ during the fall in opened- and closed-house broiler farms.

\section{Bacterial counts}

TBCs revealed highly significant increases $(p<0.01)$ in environmental and non-environmental samples collected from opened-house broiler farms (4.71 CFU/ml) compared to those from closed-house broiler farms $(4.32 \mathrm{CFU} / \mathrm{ml})$. TECs revealed highly significant increases $(p<0.01)$ in environmental and non-environmental samples collected from openedhouse $(2.87 \mathrm{CFU} / \mathrm{ml})$ compared to those from closedhouse broiler farms $(1.77 \mathrm{CFU} / \mathrm{ml})$. TSC overall means revealed highly significant increases $(\mathrm{p}<0.01)$ in environmental and non-environmental samples collected from opened-house broiler farms $(0.51 \mathrm{CFU} / \mathrm{ml})$ compared to those from closed-house broiler farms $(0.12 \mathrm{CFU} / \mathrm{ml})$. 
Table-5: Live body weight and carcass quality characteristics (Mean \pm SE) in broilers raised on different housing systems during different seasons.

\begin{tabular}{|c|c|c|c|c|c|c|}
\hline \multirow{2}{*}{$\begin{array}{l}\text { Housing } \\
\text { system }\end{array}$} & \multirow[t]{2}{*}{ LBW $/=\mathbf{g}$} & \multirow[t]{2}{*}{ Carcass wt./g } & \multicolumn{4}{|c|}{ Organs/Carcass ratio } \\
\hline & & & Liver & Spleen & Heart & Bursa \\
\hline \multicolumn{7}{|c|}{ Overall means of the housing system } \\
\hline Opened-house & $1749^{b} \pm 4.6$ & $1322^{b} \pm 5.6$ & $3.2^{\mathrm{a}} \pm 0.02$ & $0.14^{b} \pm 0.00$ & $0.90^{\mathrm{b}} \pm 0.01$ & $0.08^{\mathrm{a}} \pm 0.00$ \\
\hline Closed-house & $2074^{a} \pm 6.3$ & $1756^{a} \pm 8.0$ & $3.1^{\mathrm{b}} \pm 0.02$ & $0.18^{\mathrm{a}} \pm 0.01$ & $1.01^{\mathrm{a}} \pm 0.01$ & $0.08^{a} \pm 0.00$ \\
\hline p-value & 0.002 & 0.000 & 0.005 & 0.001 & 0.004 & 0.056 \\
\hline \multicolumn{7}{|c|}{ Overall means of the seasons } \\
\hline Summer & $1935^{c} \pm 2.5$ & $1552^{b} \pm 5.6$ & $3.2^{c} \pm 0.01$ & $0.16^{b} \pm 0.00$ & $0.86^{`} \pm 0.02$ & $0.08^{\mathrm{b}} \pm 0.00$ \\
\hline Fall & $2015^{a} \pm 3.6$ & $1590^{a} \pm 9.8$ & $3.3^{\mathrm{b}} \pm 0.02$ & $0.17^{a} \pm 0.00$ & $1.14^{\mathrm{a}} \pm 0.01$ & $0.09^{\mathrm{a}} \pm 0.01$ \\
\hline Winter & $1701^{\mathrm{d}} \pm 1.2$ & $1429^{\circ} \pm 2.3$ & $2.8^{\mathrm{d}} \pm 0.02$ & $0.12^{\mathrm{c}} \pm 0.01$ & $0.76^{d} \pm 0.02$ & $0.06^{c} \pm 0.00$ \\
\hline Spring & $1995^{b} \pm 5.6$ & $1586^{a} \pm 5.8$ & $3.4^{\mathrm{a}} \pm 0.03$ & $0.17^{a} \pm 0.00$ & $1.06^{\mathrm{b}} \pm 0.01$ & $0.09^{a} \pm 0.01$ \\
\hline $\mathrm{p}$-value & 0.005 & 0.012 & 0.001 & 0.009 & 0.006 & 0.009 \\
\hline \multicolumn{7}{|c|}{ Housing system versus seasonal variation interactions } \\
\hline \multicolumn{7}{|c|}{ Opened-house } \\
\hline Summer & $1787^{b} \pm 11.0$ & $1345^{\mathrm{c}} \pm 12.8$ & $3.2^{\mathrm{c}} \pm 0.03$ & $0.13^{c} \pm 0.00$ & $0.76^{\complement} \pm 0.01$ & $0.07 \pm 0.00$ \\
\hline Fall & $1844^{\mathrm{b}} \pm 15.1$ & $1379^{b} \pm 15.3$ & $3.4^{\mathrm{b}} \pm 0.03$ & $0.15^{b} \pm 0.00$ & $1.09^{\mathrm{a}} \pm 0.03$ & $0.08^{\mathrm{b}} \pm 0.00$ \\
\hline Winter & $1497^{\mathrm{d}} \pm 6.7$ & $1168^{\mathrm{d}} \pm 12.8$ & $2.9^{d} \pm 0.04$ & $0.10^{c} \pm 0.00$ & $0.74^{\mathrm{c}} \pm 0.01$ & $0.07 \mp 0.00$ \\
\hline Spring & $1866^{a} \pm 9.5$ & $1396^{a} \pm 11.6$ & $3.5^{\mathrm{a}} \pm 0.03$ & $0.16^{\mathrm{a}} \pm 0.00$ & $1.01^{\mathrm{b}} \pm 0.01$ & $0.09^{a} \pm 0.00$ \\
\hline p-value & 0.000 & 0.000 & 0.006 & 0.000 & 0.001 & 0.004 \\
\hline \multicolumn{7}{|l|}{ Closed-house } \\
\hline Summer & $2083^{c} \pm 6.25$ & $1758^{\mathrm{c}} \pm 8.99$ & $3.1^{\mathrm{c}} \pm 0.03$ & $0.18^{c} \pm 0.00$ & $0.96^{c} \pm 0.02$ & $0.08^{\mathrm{b}} \pm 0.00$ \\
\hline Fall & $2185^{a} \pm 5.97$ & $1801^{a} \pm 8.71$ & $3.3^{\mathrm{a}} \pm 0.04$ & $0.20^{a} \pm 0.00$ & $1.20^{\mathrm{a}} \pm 0.02$ & $0.09^{a} \pm 0.00$ \\
\hline Winter & $1905^{d} \pm 5.70$ & $1689^{d} \pm 7.45$ & $2.7^{\mathrm{d}} \pm 0.03$ & $0.13^{d} \pm 0.00$ & $0.78^{d} \pm 0.01$ & $0.05^{c} \pm 0.00$ \\
\hline Spring & $2123^{b} \pm 5.78$ & $1777^{b} \pm 8.53$ & $3.2^{b} \pm 0.03$ & $0.19^{b} \pm 0.00$ & $1.11^{\mathrm{b}} \pm 0.02$ & $0.09^{a} \pm 0.00$ \\
\hline $\mathrm{p}$-value & 0.006 & 0.000 & 0.000 & 0.000 & 0.004 & 0.009 \\
\hline
\end{tabular}

Means carrying different superscripts in the same column are significantly different at $(p \leq 0.05)$ or highly significantly different at $(p<0.01)$. Means carrying the same superscripts in the same column are non-significantly different at $(p<0.05)$. LBW=Live Bodyweight, Carcass wt=Carcass weight, opened=naturally ventilated houses, Closed=artificially ventilated houses, $\mathrm{SE}=\mathrm{Standard}$ error

TBCs revealed highly significant increases $(\mathrm{p}<0.01)$ in environmental and non-environmental samples collected during spring, winter, summer, and fall seasons $(4.57,4.50,4.49$, and $4.41 \mathrm{CFU} / \mathrm{ml}$, respectively). TECs revealed highly significant increases $(p<0.01)$ in environmental and non-environmental samples collected during summer, winter, spring, and fall seasons $(4.57,4.50,4.49$, and $4.41 \mathrm{CFU} / \mathrm{ml}$, respectively) with no significant differences between TEC during winter and spring seasons. TSCs revealed highly significant increases $(\mathrm{p}<0.01)$ in environmental and non-environmental samples collected during summer, spring, winter, and fall seasons $(0.38,0.38$, 0.36 , and $0.35 \mathrm{CFU} / \mathrm{ml}$, respectively) with no significant differences between TSCs during summer and spring seasons.

Total bacterial and Enterobacteriaceae counts interactions, in Figures-1 and 2, revealed highly significant increases $(p<0.01)$ in the litter, water, fans, feeders, waterers, swabs, liver, spleen, intestine, and breast muscles collected from opened-house broiler farms during spring, and in the wall swabs during fall and spring, respectively. Highly significant increases $(\mathrm{p}<0.01)$ were recorded in TBC and TEC of litter, water, walls, fans, feeders, waterers swabs, liver, spleen, intestine, and breast muscles of closedhouse broiler farms during summer compared to other seasons.

TSCs (Figure-3) revealed highly significant increases $(p<0.01)$ in the litter, water, walls, feeders, waterers swabs, liver, intestine, and breast muscle during spring in opened-house, and summer in closedhouse broiler farms. Meanwhile, in Figure-3, highly significant increases $(p<0.01)$ were observed during winter and summer in fan swabs, and during spring and fall in the spleen ratios of opened- and closedhouse broiler farms, respectively.

\section{Salmonella serotype and prevalence}

$S$. Typhi $\mathrm{O}$ and $\mathrm{H}$ and $S$. Paratyphi A and B titer revealed in Table- 2 highly significant increases $(p<0.01)$ in opened-house compared to closed-house broiler farms. $S$. Typhi O (Table-2) revealed highly significant increases $(\mathrm{p}<0.01)$ in summer, fall, winter, and spring, respectively, with no significant differences during summer and fall seasons and between winter and spring seasons. S. Typhi H and S. Paratyphi $\mathrm{A}$ and $\mathrm{B}$ titer revealed in Table-2 no significant differences between the four seasons.

$S$. Typhi O and H, as well as, S. Paratyphi A and B titer revealed, in Table-2, highly significant increases $(p<0.01)$ in opened-house broiler farms during summer seasons and in closed-house broiler farms during fall seasons. Salmonella spp. prevalence revealed highly significant increases $(\mathrm{p}<0.01)$ during summer, fall, winter, and spring, respectively. The calculation of the average prevalence percent of salmonellosis in both types of broiler farms understudy (Table-2) showed that the prevalence of salmonellosis in the closed-house broiler farms (6.0\%) was significantly 


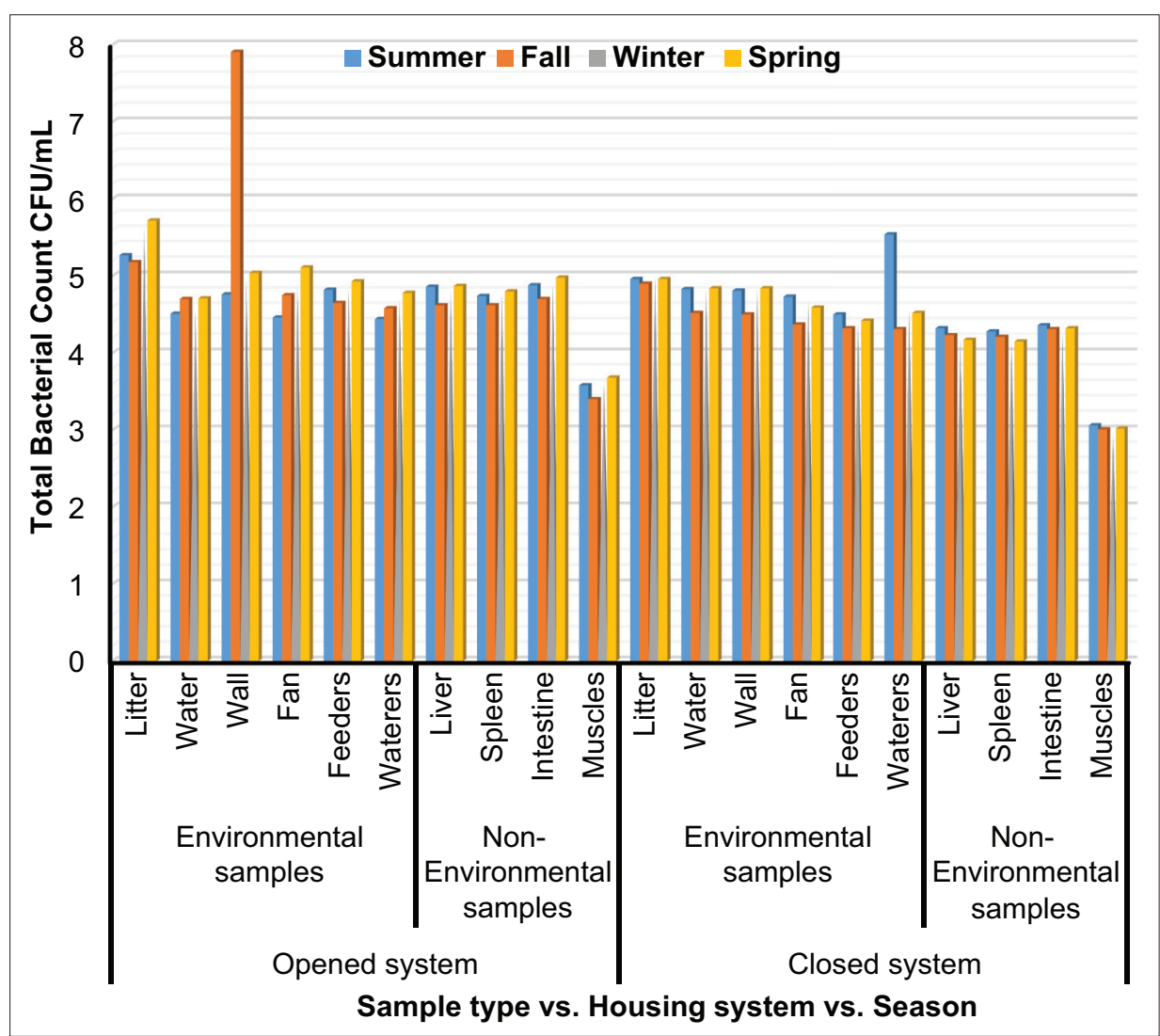

Figure-1: Logarithm total bacterial counts (Mean \pm Standard error colony-forming units/ml) in environmental and nonenvironmental samples collected from different housing systems during different seasons.

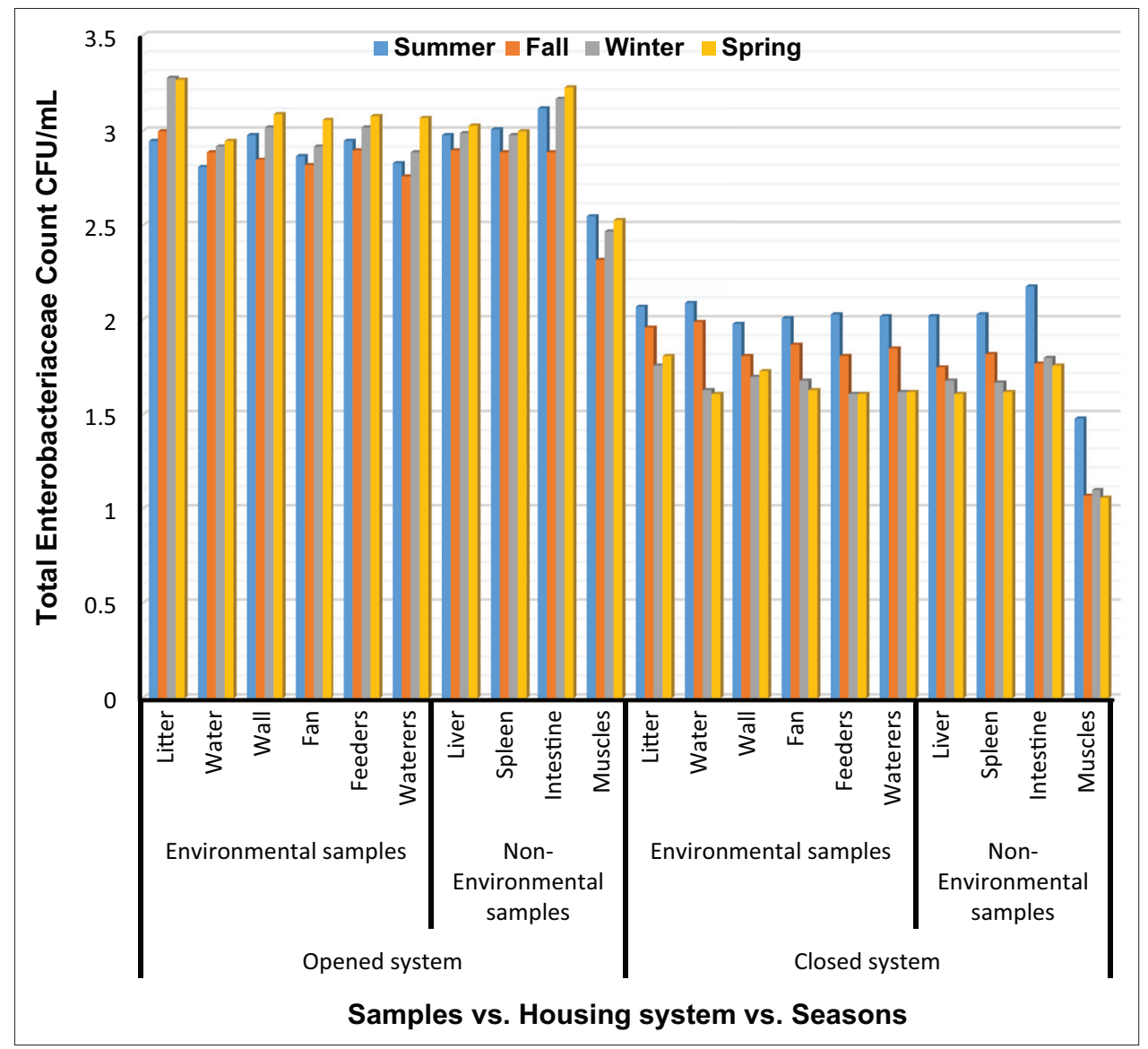

Figure-2: Logarithm total Enterobacteriaceae counts (Mean \pm Standard error colony-forming units/ml) in environmental and non-environmental samples collected from different housing systems during different seasons. 


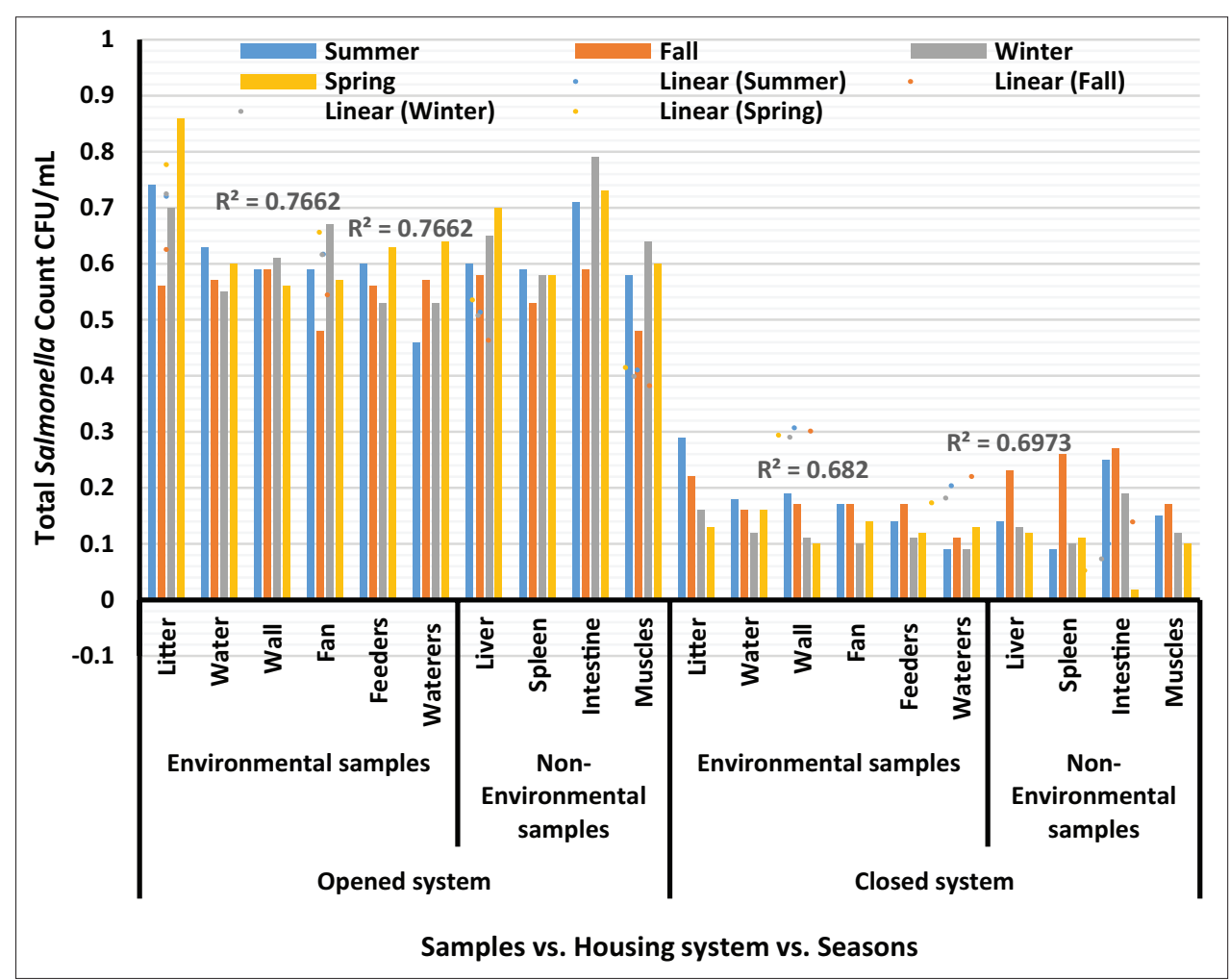

Figure-3: Logarithm total Salmonella counts (Mean \pm Standard error colony-forming units/ml) in environmental and nonenvironmental samples collected from different housing systems during different seasons.

lower $(\mathrm{p}<0.01)$ compared to that in the opened-house broiler farms $(24.67 \%)$.

\section{Biosecurity measures status}

A total score was given for each farm as a sum for all individual average scores for the listed evaluation items, as shown in Table-3. The calculated average score for the closed-house broiler farms was 34 out of 43 and for the opened-house broiler farms was 24 out of 43 . The results revealed higher levels of commitment and disciplines of biosecurity measures in closed-house compared to opened-house broiler farms.

\section{Discussion}

Poultry production worldwide and in Egypt depends on intensification in naturally ventilated (opened) and artificially ventilated (closed) housing systems associated with different floor systems such as deep litter, battery, and slatted floor systems with or without an all-in-all out policy. Broilers in the commercial and small yard production remain inside their raising or growing and finishing houses for the entire cycle, then removed for slaughtering or transferred to another egg-laying facility [28]. Chapman and Jeffers [29] classified the transportation conditions to the slaughterhouses and the movement of birds to other buildings for egg production as suitable conditions contribute to lowering broiler's resistance and creating a perfect media and circumstances that enhance the introduction and establishment of infectious agents, colonize broilers, and establish a cycle of infection unless there are enough preventive measures installed to prevent the introduction and spread of these pathogens.

Small scale and backyard poultry production in Egypt has become a profitable industry for many reasons including minimum maintenance requirements, profitable and fast financial outcome, coverage of a gap in public demand for animal protein, easy marketing, provision of high-quality fertilizer, and easy control through the application of a small number of preventive measures [30]. Total household and backyard poultry population in Egypt is undefined, and all the authorized and available governmental data referred to the financial investment sizes without exact numbers, but according to Helal et al. [31], about 9.5 million poultry are raised in small and confined places with little or no biosecurity measures. ElMasry et al. [32] reported a significant role in the backyard and household production in the spread and transmission of many infectious and zoonotic diseases.

The prevalence of salmonellosis in the current study revealed a significantly higher prevalence in the opened-house compared to closed-house broiler farms without neglecting the biosecurity measures installed in each farm, and the favorable macroclimatic condition predominated to stimulate Salmonella spp. growth and survival. Rothrock et al. [33] indicated that the poultry industry has been incriminated in many salmonellosis outbreaks among poultry and human populations worldwide with special reference to Salmonella enterica serovar Enteritis and S. enterica serovar Typhimurium. Fasanmi et al. [34] stated that the persistence of pathogens such as avian influenza 
H5N1 and Salmonella in certain geographical areas is attributed to poor biosecurity measures in the poultry farms and live bird markets.

Soliman et al. [35] have earlier conducted an epidemiological survey and isolated Escherichia coli, Salmonella spp., Proteus vulgaris, Shigella spp., Pseudomonas aeruginosa, and Streptococcus faecalis from 416 environmental samples collected during the period January 2008-July 2008 from commercial poultry farms in Ismailia and Zagazig governorates Egypt, and from 266 environmental and non-environmental samples collected during the period September 2008-January 2009 by Alabama State Veterinary Diagnostic Laboratory - USA. They attributed these large proportions of microbial contaminants to the lack of sufficient hygienic and biosecurity measures in these farms. Our results were not consistent with those recorded by Wierup et al. [36] who compared the occurrence of salmonellosis during 2007-2015 in broiler and laying hen in outdoor and indoor production under the influence of the synchronized conditions for prevention and control of Salmonella spp. in Sweden. They found a very small annual incidence of Salmonella spp. in outdoor production (0-2.0\%) similar to that of indoor production (0-1.3\%), they attributed this presence to the horizontal and vertical transmission means of Salmonella spp.

$S$. Typhi $\mathrm{O}$ and $\mathrm{H}$ detection with higher prevalence in opened-house broiler farms in our study might be attributed to the misapplication of biosecurity main disciplines as disinfection procedures. Results agreed with those of Ahmed et al. [37] who investigated the efficiency of 125 and $250 \mathrm{ml}$ chlorine dioxide 2000 ppm against E. coli and Salmonella spp. They revealed a significant reduction in both types of microorganisms in normal microclimatic conditions.

Biosecurity has been defined as "informed common sense," meaning obligations to learn principles and regulations of biosecurity, and mixed with common management practices. Discipline, accountability, and belief are the main three pillars on which the biosecurity program relies. The final average scores calculated in the current study for each of the nine farms implemented an average of 34 out of 43 for closed-house and 24 out of 43 for opened-house broiler farms, meaning higher commitment in closed-house broiler farms toward the application of biosecurity measures compared to opened-house broiler farms. The results were synchronized with those recorded by Aiyedun et al. [38] who investigated biosecurity status in some broiler farms depending on the presence of a fence, traffic signals, dead bird disposal methods, usage of protective clothes, access of wild birds, and rodents. They explained that the misapplication of basic hygienic and biosecurity measures contributed to serious consequences on birds' and human health.

In the current study, cleaning and disinfection programs were observed to ensure applying biosecurity measures announced on each farm. Dry cleaning was applied to remove loose dirt, followed by wet cleaning using detergent and water under pressure, with paying attention for corners, joints, and fissures in walls and floors. The most predominating detergents that were used in the nine farms were quaternary ammonium compounds or a mixture of aldehydes and quaternary ammonium compounds. Disinfection processes were carried out using sodium hydroxide followed by formaldehyde spray in opened-house broiler farms, and the same procedures were used in addition to sodium hypochlorite as an additional step in closedhouse broiler farms. Cleaning and disinfection procedures in some farms understudy were effective to minimize the entrance of Salmonella spp. The results are in agreement with those of Klosha et al. [39] who reported that the application of risk-oriented hygiene contributed minimization of public health risk from Salmonella spp., and $66 \%$ reduction in salmonellosis incidence can be achieved through intensive cleaning. Soliman et al. [40] evaluated the disinfection regimens in some commercial poultry farms located in Ismailia and Zagazig governorates - Egypt. They recovered some bacterial microorganisms such as Klebsiella oxytoca, Citrobacter spp., P. vulgaris, E. coli, $P$. aeruginosa, Shigella spp., Salmonella spp., $S$. faecalis, Staphylococcus aureus, and Streptococcus pneumoniae, and some fungal organisms such as Yeast spp., Candida albicans, Aspergillus flavus, Aspergillus niger, Aspergillus nidulans, Mucor, and Penicillium spp. They attributed the recovery of these organisms to the failure of disinfection regimens that were followed in these farms.

The recorded results were also consistent with those of Luyckx et al. [41], who evaluated the efficacy of four cleaning programs to reduce $E$. coli and Salmonella spp. infection in 12 broiler houses. They recorded a significant reduction in total aerobic flora and Enterococcus spp. counts in swabs after the cleaning process with no differences between protocols. Course [42] investigated disinfection status as a key role in biosecurity program in the commercial broiler farms in Ontario, and he revealed that efficient disinfection procedures can be detrimental for many pathogens such as E. coli, Clostridium perfringens, and $S$. enterica. Soliman et al. [43] found that cleaning and disinfection procedures using the recommended disinfectants' concentration were not able to control microbial growth and subsequent contamination with some zoonotic enteric pathogens such as E. coli and Salmonella spp.

Our results agreed with those recorded by Limbergen et al. [44], who quantified the biosecurity levels in broiler farms using a risk-based weighted scoring system, consisted of an external scoring system with eight subcategories and internal scoring system with three subcategories. They reported that broiler farms had internal biosecurity 76.6 and external biosecurity 68.4; the results indicated the presence of wide variation for both levels of biosecurity, and 
the great possibility for improving biosecurity level of each farm.

\section{Conclusion}

Biosecurity measures were enforced with a higher degree of success in artificially ventilated (closed-house) compared to naturally ventilated (opened-house) broiler farms. Needless to say, installing strict biosecurity measures in both types of broiler houses were not enough to prevent the growth and multiplication of Salmonella spp. The lack of commitment to the adopted measures contributed a breach through which microorganisms can enter, stabilize themselves, develop infectious and contagious diseases, and spread from one area to another inside the same farm or to other farms easily.

Control of salmonellosis in broiler farms regardless of the housing system, location, holding capacity, and ventilation system depend mainly on strict measures including good hygienic measures, early detection, and effective cleaning and disinfection program.

\section{Authors' Contributions}

ESS designed the cross-sectional study and sampling design, visited farms weekly during the study period, scored each farm according to the installed measures, collected samples, participated in samples analysis, interpreting results, and in writing of the manuscript. MSA participated in sample analysis, interpreting results, and writing of the manuscript.

\section{Acknowledgments}

The authors are thankful to all the farms (El-Farog, Ismailia, and Suez companies) for allowing us to collect this wide range of samples and for their patience for hearing our review on their biosecurity programs. The study was funded by the authors.

\section{Competing Interests} interests.

The authors declare that they have no competing

\section{Publisher's Note}

Veterinary World remains neutral with regard to jurisdictional claims in published institutional affiliation.

\section{References}

1. Gussem, M.D., Meddelkoop, K.V., Mullen, K.V. and Veer, E.V. (2013) Broiler Signals: A Practical Guide for Broiler Focused Management. Rood Bont Publishers BV, Netherlands.

2. Biosecurity Act. (2015) New South Wales Biosecurity Manual, Proposed Biosecurity Regulation. NSW Department of Primary Industries Biosecurity and Food Safety, New South Wales.

3. Aengwanich, W., Boonsorn, T. and Srikot, P. (2014) Intervention to improve biosecurity system of poultry production clusters (PPCs) in Thailand. Agriculture, 4(3): 231-238.

4. Sayeed, M.A., Smallwood, C., Imam, T., Mahmud, R., Hasan, R.B., Hasan, M., Anwer, M.S., Rashid, M.H. and
Hoque, M.A. (2017) Assessment of hygienic conditions of live bird markets on avian influenza in Chittagong metro, Bangladesh. Prev. Vet. Med., 142 (July 2017): 7-15.

5. Conan, A., Goutard, F.A., Sorn, S. and Vong, S. (2012) Biosecurity measures for backyard poultry in developing countries: A systematic review. BMC Vet. Res., 8 (December 2012): 240

6. Rajagopal, R. and Mini, M. (2013) Outbreaks of salmonellosis in three different poultry farms of Kerala, India. Asian Pac. J. Trop. Biomed., 3(6): 496-500.

7. Eltholth, M.M., Mohamed, R.A., Elgohary, F.A. and Elfadl, E.A. (2016) Assessment of biosecurity practices in broiler chicken farms in Gharbia governorate, Egypt. Alex. J. Vet. Sci., 49(1): 68-77.

8. Dar, M.A., Ahmad, S.M., Bhat, S.A. and Ahmed, R. (2017) Salmonella Typhimurium in poultry: A review. Worlds Poult. Sci. J., 73(2): 345-354.

9. Bhunia, A.K. (2018) Food Borne Microbial Pathogens: Mechanisms and Pathogenesis. ${ }^{\text {nd }} \mathrm{ed}$. Springer Science and Business Media, LLC, United States of America.

10. Nirmala, T.V., Reddy, A.D., Sree, E.K., Subbaiah, K.V., Raju, G.S. and Reddy, R.V.S. (2018) Salmonellosis in poultry: A case report. Int. J. Curr. Microbiol. Appl. Sci., 7(2): 2347-2349.

11. Nwabor, O.F., Dickson, I.D. and Ajibo, Q.C. (2015) Epidemiology of Salmonella and salmonellosis. Int. Lett. Nat. Sci., 47(September 2015): 54-73.

12. Zhang, S., Yin, Y., Jones, M.B., Zhang, Z., Kaiser, B.L.D., Dinsmore, B.A., Fitzgerald, C., Fields, P.I. and Deng, X. (2015) Salmonella serotype determination utilizing high-throughput genome sequencing data. J. Clin. Microbiol., 53(5): 1685-1692.

13. Bellhouse, D.R. (2014) Systematic Sampling Methods. Encyclopedia of Biostatistics. Wiley StatsRef: Statistics Reference Online, New York.

14. Soliman, E.S. and Hassan, R.A. (2019) Impact of lighting color and duration on productive performance and Newcastle disease vaccination efficiency in broiler chickens. Vet. World, 12(7): 1052-1059.

15. National Research Council. (1994) Nutrient Requirements for Poultry. National Research Council, New York.

16. Applegate, T.J. and Angel, R. (2014) Nutrient requirements of poultry publication: History and need for an update. $J$. Appl. Poult. Res., 23(3): 567-575.

17. Wei, H. and Aengwanich, W. (2012) Biosecurity evaluation of poultry production cluster (PPCs) in Thailand. Int. J. Poult. Sci., 11(9): 582-588.

18. Soliman, E.S., Hamad, R.T. and Ahmed, A. (2017) Prophylactic and immune-modulatory influences of Nigella sativa Linn. in broilers exposed to biological challenge. Vet. World, 10(12): 1447-1455.

19. Thrusfield, M. (2005) Veterinary Epidemiology. $3^{\text {rd }}$ ed. Oxford: Wiley Blackwell. p228-330.

20. Soliman, E.S. and Hassan, R.A. (2017) Evaluation of superphosphate and meta-bisulfite efficiency in litter treatment on productive performance and immunity of broilers exposed to ammonia stress. Adv. Anim. Vet. Sci., 5(6): 253-259.

21. Jesudason, M.V., Sridharan, G., Arulselvan, R., Babu, P.G. and John, T.J. (1998) Diagnosis of typhoid fever by the detection of anti-LPS and anti-flagellin antibodies by ELISA. Indian J. Med. Res., 107(May 1998): 204-207.

22. Wain, J. and Hosoglu, S. (2008) The laboratory diagnosis of enteric fever. J. Infect. Dev. Ctries., 2(6): 421-425.

23. Soliman, E.S., Sallam, N.H. and Abouelhassan, E.M. (2018) Effectiveness of poultry litter amendments on bacterial survival and Eimeria oocyst sporulation. Vet. World, 11(8): 1064-1073.

24. American Public Health Association, American Water Works Association, Water Environment Federation. (2012) Standard Methods for the Examination of Water and Wastewater. $22^{\text {th }}$ ed. American Water Works Association Publications, Washington, DC. 
25. Kim, S.K. and Lee, J.H. (2016) Biofilm modeling systems. Korean J. Microbiol., 52(2): 125-139.

26. Murray, P.R., Rosenthal, K.S. and Pfaller, M.A. (2015) Medical Microbiology. $8^{\text {th }}$ ed. Elsevier Health Sciences, Philadelphia, PA, USA.

27. Green, S.B. and Salking, N.J. (2016) Using SPSS for Windows and Macintosh, Books a la Carte. $8^{\text {th }}$ ed. Pearson $\mathbb{C}$, London, United Kingdom.

28. Sharma, D., Singh, N.K., Singh, H., Joachim, A., Rath, S.S. and Blake, D.P. (2018) Discrimination, molecular characterization and phylogenetic comparison of porcine Eimeria spp. in India. Vet. Parasitol., 255(May 2018): 43-48.

29. Chapman, H.D. and Jeffers, T.H. (2014) Vaccination of chickens against coccidiosis ameliorates drug resistance in commercial poultry production. Int. J. Parasit., 4(3): 214-217.

30. BAHS. (2015) Annual Report, 2014-2015.

31. Helal, A.M., Arafa, A., Abdien, H.F., Hamed, D.M. and ElDimerdash, M.Z. (2017) Avian influenza in liver bird markets in the Suez Canal Region, Egypt. Zagazig Vet. J., 45(4): 340-348.

32. ElMasry, I., Elshiekh, H., Abdlenabi, A., Saad, A., Arafa, A., Fasina, F.O., Lubroth, J. and Jobre, Y.M. (2017) Avian influenza H5N1 surveillance and its dynamics in poultry in live bird markets, Egypt. Transbound. Emerg. Dis., 64(3): 805-814.

33. Rothrock, M.J. Jr., Ingram, K.D., Gamble, J., Guard, J., Cicconi-Hogan, K.M., Hinton, A. Jr. and Hiett, K.L. (2015) The characterization of Salmonella enetrica serotypes isolated from the scalder tank water of a commercial poultry processing plant: Recovery of a multidrug-resistant Heidelberg strain. Poult. Sci., 94(3): 467-472.

34. Fasanmi, O.G., Odetokun, I.A., Balogun, F.A. and Fasina, F.O. (2017) Public health concerns of highly pathogenic avian influenza H5N1 endemicity in Africa. Vet. World, 10(10): 1194-1204.

35. Soliman, E.S., Reddy, P.G., Sobieh, M.A.A., Busby, H. and Rowe, S. (2009) Epidemiological surveillance on environmental contaminants in poultry farms. Int. J. Poult. Sci., 8(2): 151-155.
36. Wierup, M., Wahlström, H., Lahti, E., Eriksson, H., Jansson, D.S., Odelros, A. and Ernholm, L. (2017) Occurrence of Salmonella spp.: A comparison between indoor and outdoor housing of broilers and laying hens. Acta Vet. Scand., 59 (February 2017): 13.

37. Ahmed, S.T., Bostami, A.B.M., Mun, H.S. and Yang, C.J. (2017) Efficacy of chlorine dioxide gas in reducing Escherichia coli and Salmonella from broiler house environments. J. Appl. Poult. Res., 26(1): 84-88.

38. Aiyedun, J.O., Oludairo, O.O., Olorunsola, I.D., Daodu, O.B. and Furo, N.A. (2018) Effectiveness of biosecurity measures in some selected farms in Kwara State, Nigeria. J. Res. For. Wildl. Environ., 10(2): 17-23.

39. Klosha, F., Casteel, M., Kump, F.W. and Klein, G. (2017) Implementation of a risk-orientated hygiene analysis for the control of Salmonella JAVA in the broiler production. Curr. Microbiol., 74(3): 356-364.

40. Soliman, E.S., Sobieh M.A.A., Ahmad, Z.H., Hussein, M.M., Abdel-Latiff, H. and Moneim, A.A. (2009) Seasonal epidemiological surveillance on bacterial and fungal pathogens in broiler farms in Egypt. Int. J. Poult. Sci., 8(8): 720-727.

41. Luyckx, K.Y., Van Weyenberg, S., Dewulf, J., Herman, L., Zoons, J., Vervaet, E., Heyndrickx, M. and De Reu, K. (2015) On-farm comparisons of different cleaning protocols in broiler houses. Poult. Sci., 94(8): 1986-1993.

42. Course, C.E. (2018) Factors Associated with Salmonella enterica, Escherichia coli, and Clostridium perfringens in Broiler Barns during Downtime. Master Thesis. Ontario, Canada: University of Guelph.

43. Soliman, E.S., Moawed, S.A. and Ziaan, A.M.G. (2016) Assessing cleaning and disinfection regime in a slaughterhouse against carcasses contamination. Adv. Anim. Vet. Sci., 4(9): 449-457.

44. Limbergen, T.V., Dewulf, J., Klinenberg, M., Ducatelle, R., Gelaude, P., Mendez, J., Heinola, K., Papasolomontos, S., Szeleszczuk, P. and Maes, D. (2017) Scoring biosecurity in European conventional broiler production. Poult. Sci., 97(1): 74-83.

$* * * * * * * *$ 